\title{
An intelligent fuzzy logic controller applied to multi-area load frequency control
}

\author{
Ndubisi Samuel N \\ Department of Electrical and Electronic Engineering,
Enugu State University of Science and Technology, P.M.B. 01660 Enugu - Nigeria.
}

\section{ABSTRACT}

Continuous load perturbation in power systems network affects the frequency directly. If the system load frequency control (LFC) mechanism is working perfectly, the frequency is quickly returned to the normal operating point. Many types of controllers have been employed for the LFC but due to non linearity of power system components and generators, the response are not usually fast and efficient. The fuzzy logic controller which is based on human expertise knowledge is employed in this paper to overcome all the setbacks witnessed in all the other controllers. The result shows an improved performance in terms of rise time, settling time, steady state error and overshoot.

\section{Keywords: Area control error, Tie-lic
fuzzy logic controller, Bias factor. INTRODUCTION}

In interconnected power system, there are two major controls installed in each generator, the load frequency control (LFC) and the automatic voltage regulator (AVR). The LFC which is the primary focus of this paper is to maintain uniform frequency, divide the load between generators and to control the tieline interchange schedules [1]. The various areas of the power system network are connected with tie lines. The tie-lines are utilized for contractual energy exchange between areas and provide inter-area support in abnormal conditions. Area load changes and abnormal conditions due to system fault (resulting to generation outages) results to mismatch in frequency and scheduled power interchanges between areas. When there is mismatch, it must be controlled through supplementary control. Primarily, the load frequency control of interconnected systems is defined as the regulation of power output of generators within a prescribed area, in response to change in frequency, tie-line loading, or the relation of these to each other, so as to maintain scheduled system frequency and/or establish interchange with other areas within predetermined limits. A lot of research reports have been made on LFC controls of interconnected power systems, all geared toward control strategies which will better the design of load frequency controllers in order to achieve better dynamic performance. The most applied load frequency controllers is the conventional proportional integral (PI) controller [3,6]. The PI controllers are easier to implement but usually gives large frequency deviation and large settling time depending on the turning of the constants. Linear optimal control theory employing state feedback controllers have been proposed for enhanced performance [7, 8, 9]. In order to overcome the set backs of fixed gain controllers which are usually designed to operate at nominal conditions and which fails to provide control over a wide range of operating conditions, adaptive self tuning controllers which usually tracks the operating conditions and use updated parameters to compute the control are proposed for LFC $[10,11]$. The drawbacks of adaptive controllers are the complicated control algorithm and the on-line system modal identification. Most researches going on now is based on artificial intelligent systems (fuzzy and neural networks). The inherent gain of these techniques is that they do not require the system model and identification but depends on human expertise knowledge of the behaviour of the LFC problem [3, 12, 13]. In this paper, a fuzzy PI controller is proposed and performance comparison is carried out for conventional PI, optimal controllers and fuzzy PI LFC. The simulation result shows improved system performance when the fuzzy PI controller is employed even outside the operating range.

\section{POWER SYSTEM MODELING FOR LFC CONTROL:}

Single Area System with Conventional PI Controller: The block diagram of fig. 1 shows a load frequency controlled scheme using conventional PI controller for an isolated power system. Primarily, the task of LFC is to generate a control signal that 
maintains system frequency and tie-line interchange power at predetermined values. A transfer function approach is used to obtain the overall transfer function as
Appendix A shows the values of parameters of an isolated power station. Figure 2 shows the response of the power station for sudden load drop of $\Delta P_{L}=0.2$ per unit and for values of $K_{l}$ from 1 to 9 .

$$
\frac{\Delta w(s)}{-\Delta P_{L}(s)}=\frac{s\left(1+\tau_{q} s\right)\left(1+\tau_{T} s\right)}{s(2 H s+D)\left(1+\tau_{q} s\right)\left(1+\tau_{T} s\right)+K_{I}+S / R}
$$

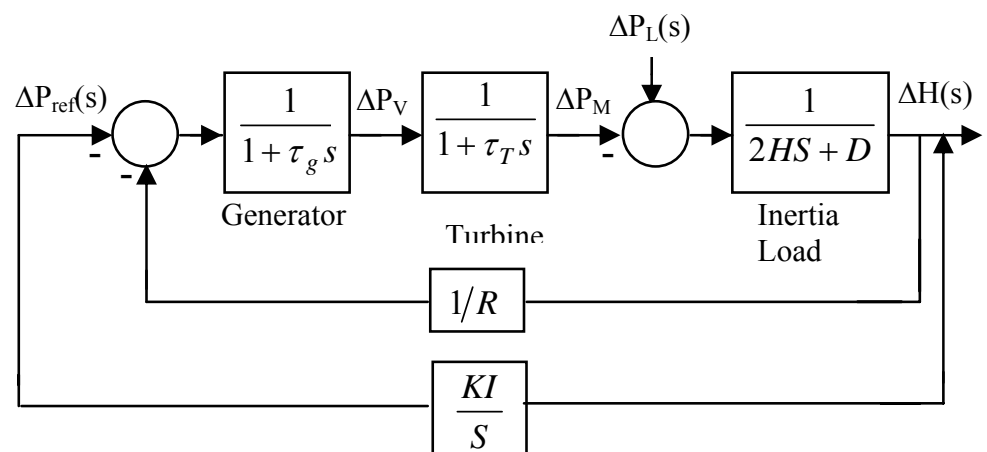

Fig.1: PI controller for automatic gain control (AGC) of power system

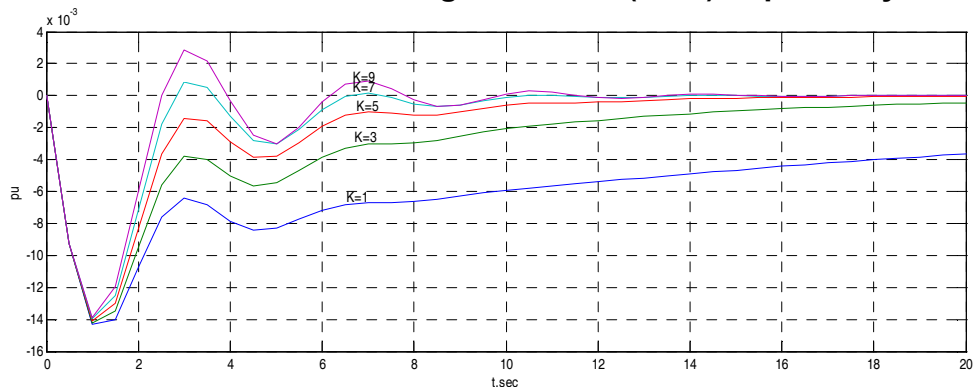

Fig. 2: Frequency Response of the system for sudden change of $\Delta P_{L}$ and for different values of $K_{I}$

\section{Modelling of a multi area power system LFC with conventional PI controller}

Fig. 3 below refers to equivalent diagram of two coherent groups of generators in a control area. Let $\mathrm{X}_{\text {tie }}$ be reactance of a lossless tie-line with each generator represented by voltage source behind an equivalent reactance. During normal operation, the real power transfer over the tie -line is

$$
P_{12}=\frac{\left|E_{1} \| E_{2}\right| \sin \partial_{12}}{X_{12}}
$$

where $X_{12}=X_{1}+X_{\text {tie }}+X_{2}$, and $\delta_{12}=\delta_{1}-\delta_{2}$. Linearizing $\mathrm{P}_{12}$ for small deviation in tie line flow,

$$
\Delta P_{12}=\frac{d P_{12}}{d \delta_{12}} / \delta_{120}
$$

Let

$$
\Delta P_{12}=\frac{d P_{12}}{d \delta_{12}}=\frac{\left|E_{1} \| E_{2}\right| \cos \Delta \delta_{120}}{X_{12}}
$$

$$
=\text { synchronizing power coefficient }
$$

Where $\delta_{120}=\delta_{10}-\delta_{20}=$ initial operating angle. Equation (4) becomes,

$$
\Delta P_{12}=P_{s}\left(\Delta \delta_{1}-\Delta \delta_{2}\right)
$$

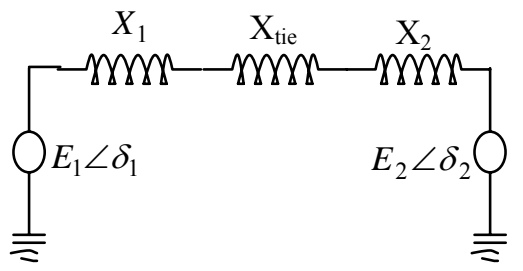

Fig. 3: Equivalent network for 2-area power system 
Am. J. Sci. Ind. Res., 2010, 1(2): 220-226

If there is load change $\Delta P_{L 1}$, in area 1 , in steady state both areas will have the same steadystate frequency deviation given by

$$
\Delta w=\Delta w_{1}=\Delta w_{2}
$$

and

$$
\begin{aligned}
& \Delta P_{m 1}-\Delta P_{12}-\Delta P_{L 1}=\Delta w \Delta_{1} \\
& \Delta P_{m 2}+\Delta P_{12}=\Delta w \Delta_{2}
\end{aligned}
$$

The change in mechanical power determined by the governor speed characteristics is given by

$$
\begin{aligned}
& \Delta P_{m 1}=\frac{-\Delta w}{R_{1}} \\
& \Delta P_{m 2}=\frac{-\Delta w}{R_{2}}
\end{aligned}
$$

From (8) and (9),

$$
\Delta w=\frac{-\Delta P_{L 1}}{\left(\frac{1}{R_{1}}+D_{1}\right)+\left(\frac{1}{R_{2}}+D_{2}\right)}
$$

where $B_{1}=\frac{1}{R_{1}}+D_{1}$

$$
B_{2}=\frac{1}{R_{2}}+D_{2}
$$

$\mathrm{B}_{1}$ and $\mathrm{B}_{2}$ are the frequency bias factors. The change in tie-line power is

$$
\Delta P_{12}=\frac{-B_{2} \Delta P_{L 1}}{B_{1}+B_{2}}
$$

Figure 4 below shows two-area system with only primary LFC loop.

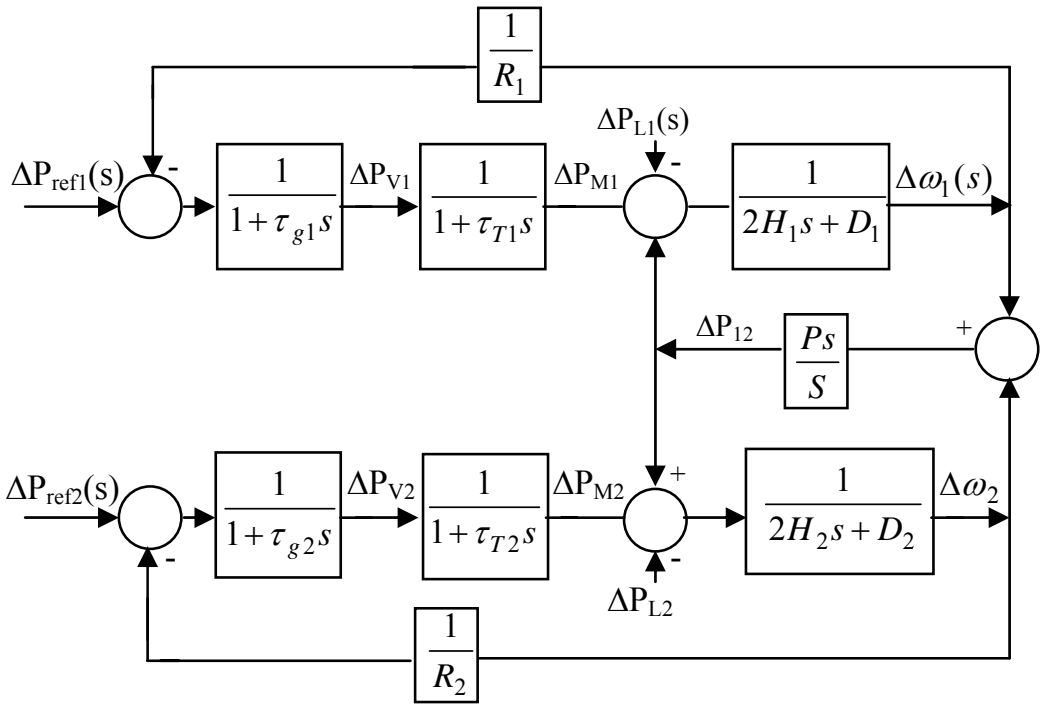

Fig. 4: Two-area system with only primary LFC loop.

\subsection{1: The tie-line bias control.}

Unlike the LFC of fig.4 which has no supplementary control mechanisms, (simulation results) the normal mode control (with supplementary control) offers the following;

- Keep frequency approximately at the nominal value $(50 \mathrm{~Hz})$

- Maintain the tie-line flow at about schedule
- Each area should absorb its own load changes.

A conventional PI LFC bases its control on tie-line bias, with each area tending to reduce each area control error (ACE) to zero. The ACE for each is given by

$$
A C E_{i}=\sum_{j=i}^{n} \Delta P_{i j}+K_{i} \Delta w
$$


Am. J. Sci. Ind. Res., 2010, 1(2): 220-226

where $\mathrm{K}_{\mathrm{i}}$ is chosen such that $K_{1}=B_{1}=\frac{1}{R_{i}}+D_{i}$ for satisfactory operation. So for two area

$$
\begin{aligned}
& A C E_{1}=\Delta P_{12}+B_{1} \Delta w_{1} \\
& A C E_{2}=\Delta P_{21}+B_{2} \Delta w_{2}
\end{aligned}
$$

Figure 5 shows PI based block diagram for a twoarea system.

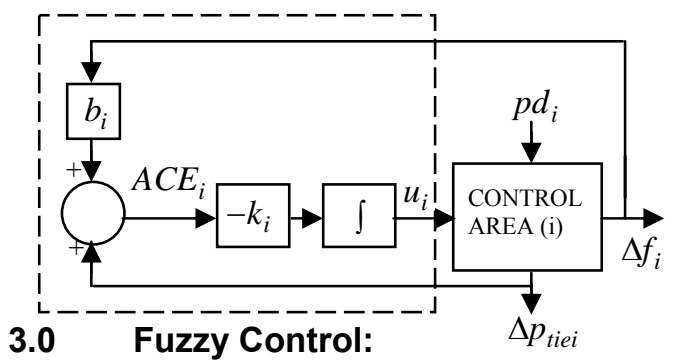

Fig. 5: Conventional PI Controller Installed on $\mathrm{i}^{\text {th }}$ Area

The application of artificial intelligent controllers (example fuzzy logic controllers) to LFC problems in power systems is now the recent trend in power system controls. This is due to numerous advantages inherent in this mode of control. The fuzzy logic control works on rule base formulated by an expert. It neglects system modelling dynamics and takes care of system non-linearities and performance outside the operating region. Hisu et.al [13] proposed a fuzzy logic controller for LFC using frequency deviation and rate as inputs. Indulkar et.al [14] proposed a controller with area control error as inputs. This work proposes a fuzzy integral controller with up to 49 rules for the control of Area control error (ACE). There are two controllers, fuzzy ACE1 and fuzzy ACE2 as shown in figure B below. Each of the controllers has the Negative Medium NM, Negative Small NS, Zero ZE, Positive Small PS, Positive Medium PM, Positive Big PB for both the Area control error and change in area control error ACE [15]. Table 1 below shows the rules. The rules are interpreted as follows.

If $A C E$ is NB and $A C E$ is NS then the output is PM.

Triangular membership functions are used for both the inputs and output. The Defuzzification method employed is the center of area method $[14,16]$
Table 1 Fuzzy Control Rules

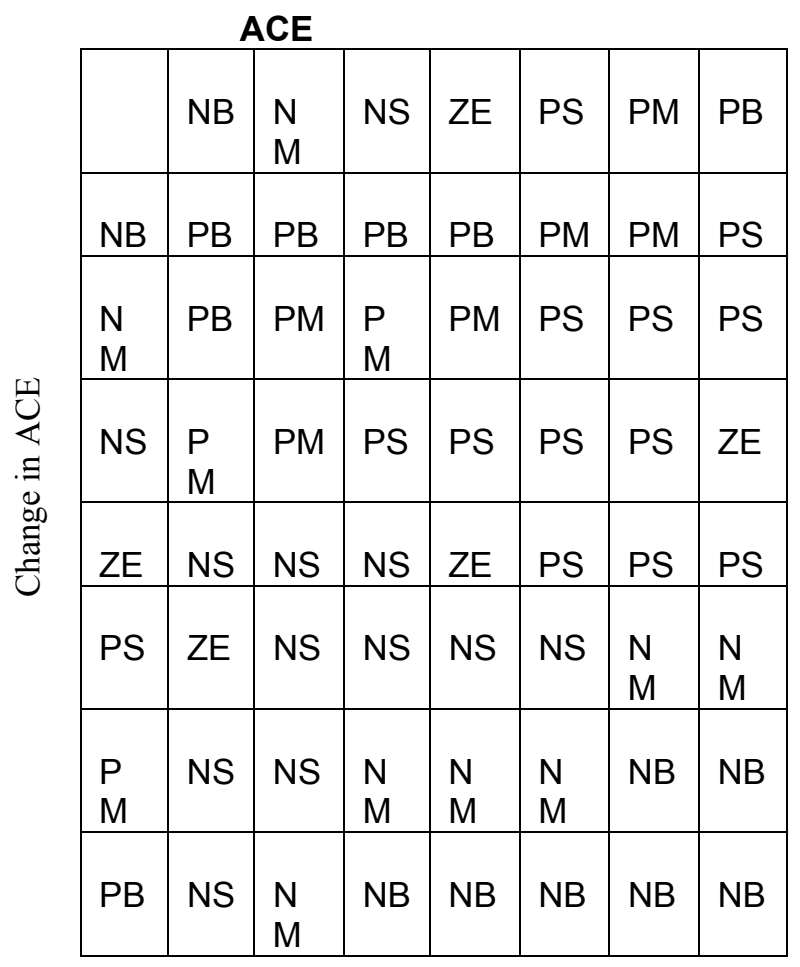

\section{SIMULATIONS AND RESULTS}

The following simulations were performed in order to investigate the performance of the proposed fuzzy logic controller over the conventional integral controller.

4.1: A two-area system connected by a tie-line with the parameters as indicated in appendix $B$ and fig. 4 for a $2 \%$ changes in load. Simulation was carried out for 25 seconds to determine the response of $\Delta \mathrm{w}_{1}, \Delta \mathrm{w}_{2}, \mathrm{Pm}_{1}, \mathrm{Pm}_{2}$ and $\mathrm{P}_{12}$ (see fig. $7 \mathrm{a}, \mathrm{b})$ without supplementary controllers.

4.2: Using the same data as in 4.1 and fig. 5, now employing $\mathrm{PI}$ controllers, the simulations were repeated as shown in fig. 8 a, b with 2 and $20 \%$ changes in load.

4.3: Based on the same data and fig. 6, employing fuzzy controllers, the same simulations were repeated and results shown in fig. 9a, b. 
Am. J. Sci. Ind. Res., 2010, 1(2): 220-226
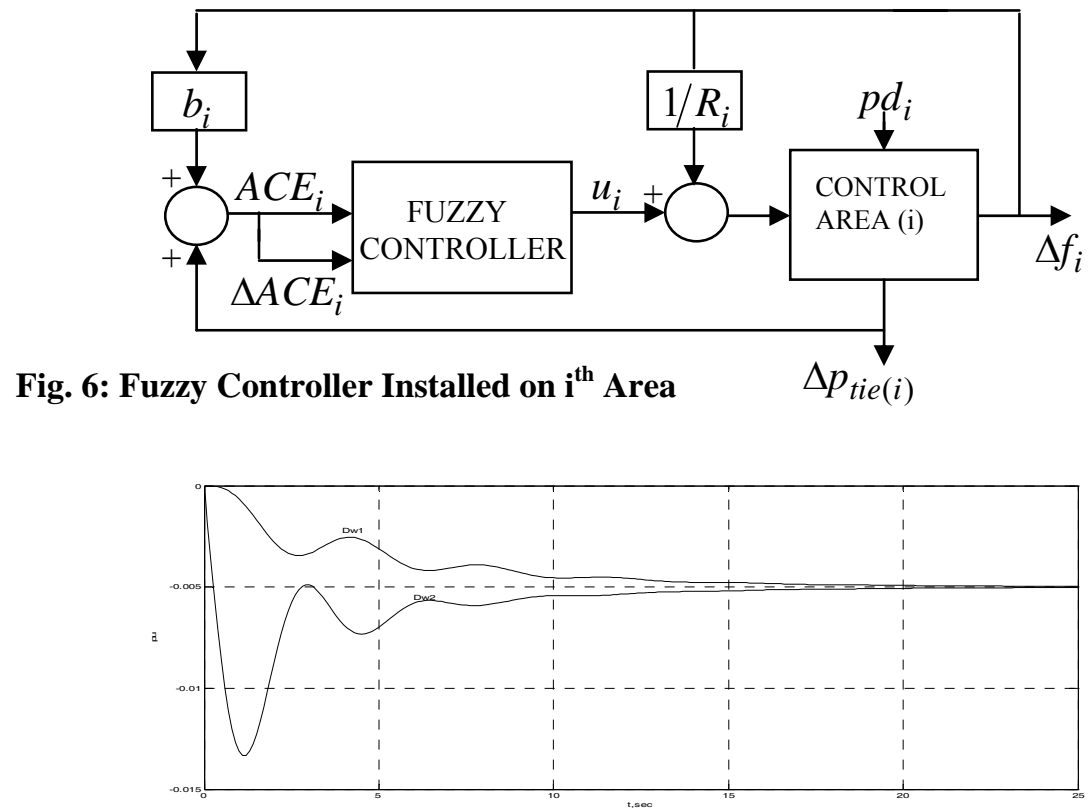

(a) Frequency deviation response for $2 \%$ change in PL.

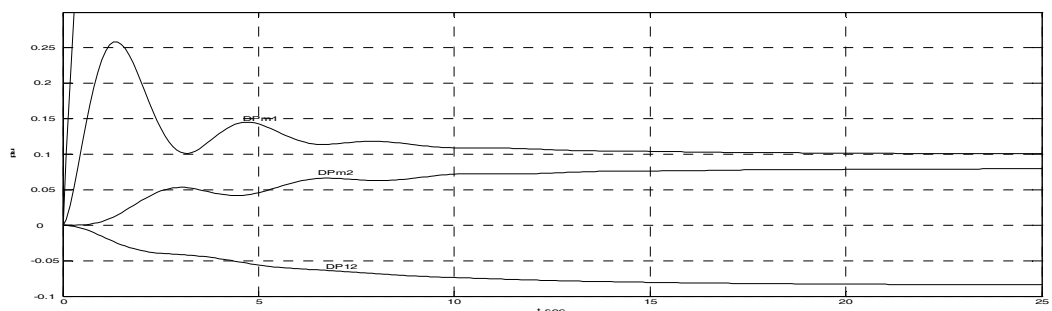

(b) Power deviation response.

Fig. 7: Response of two-area LFC without supplementary control.

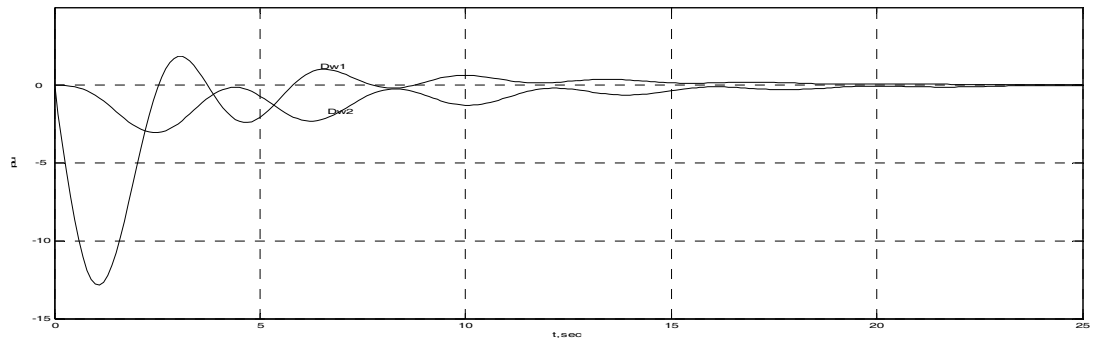

(a) Frequency deviation response for $2 \%$ change in $\mathrm{PL}$ 
Am. J. Sci. Ind. Res., 2010, 1(2): 220-226

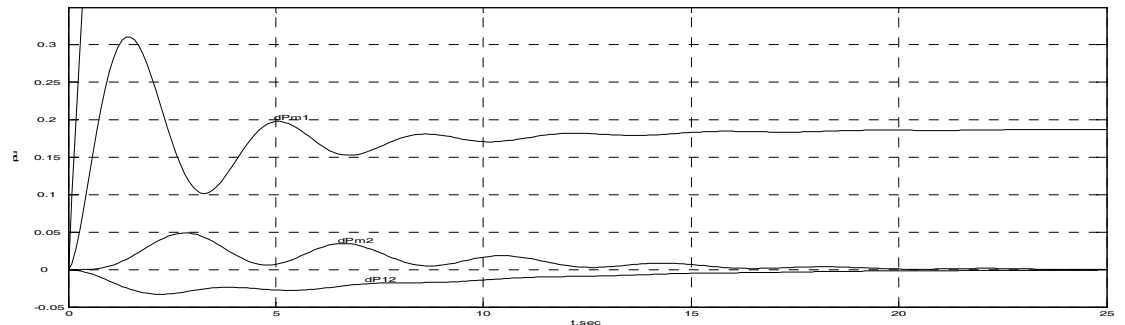

(b) Power deviation response.

Fig. 8: Response of two-area LFC with PI controllers.

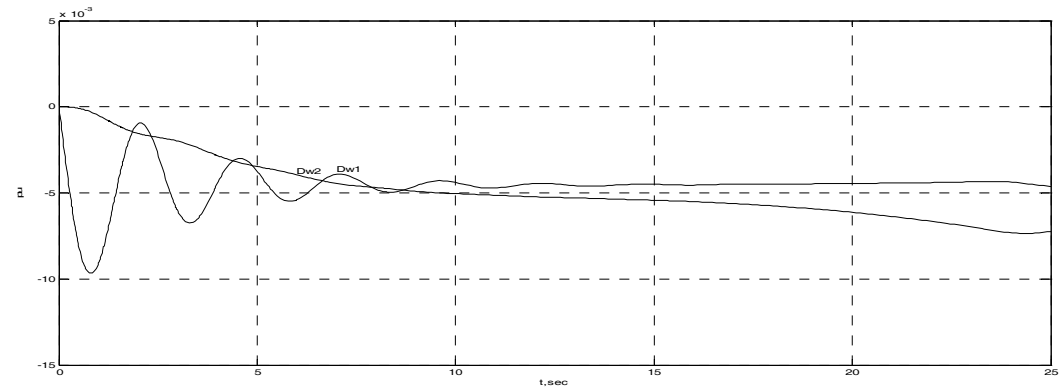

(a) Frequency deviation response for $2 \%$ change in $\mathrm{PL}$

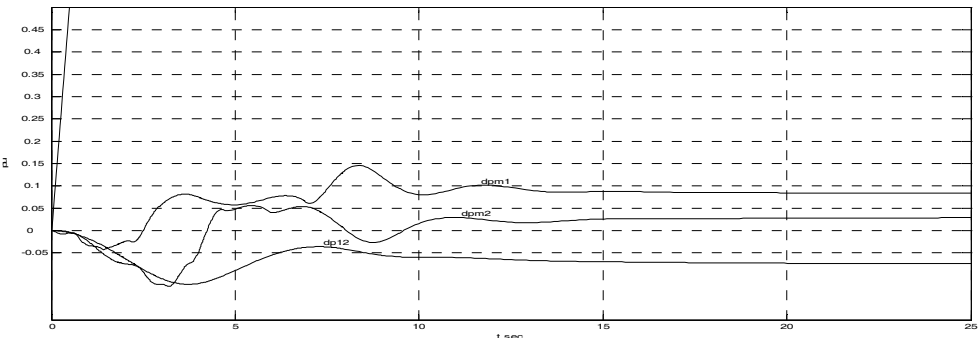

(b) Power deviation response

Fig. 9: Response of two-area LFC with Fuzzy Controller.

Table 2(a) and (b) below shows the summary of the estimated performance indices for the three cases considered

Table 2(a): SUMMARY OF FREQUENCY DEVIATION DURING LOAD CHANGE

\begin{tabular}{|l|ll|lc|lc|}
\hline TYPE OF & \multicolumn{2}{|c|}{$\%$ Overshoot } & \multicolumn{2}{|c|}{ Settling Time (sec) } & \multicolumn{2}{|c|}{ Steady state error (pu) } \\
CONTROLLER & \multicolumn{2}{|c|}{$\Delta \mathrm{F}_{1}$} & $\Delta \mathrm{F}_{2}$ & $\Delta \mathrm{F}_{1}$ & $\Delta \mathrm{F}_{2}$ & $\Delta \mathrm{F}_{2}$ \\
\hline Without controller & -0.30 & -1.3 & 22 & 22 & -0.005 & -0.005 \\
\hline With PI controller & -1.5 & -0.25 & 20 & 20 & 0.00 & 0.00 \\
\hline With Fuzzy controller & -0.09 & -0.05 & 15 & 15 & -0.005 & -0.005 \\
\hline
\end{tabular}

Table 2(b): SUMMARY OF POWER DEVIATION DURING LOAD CHANGE

\begin{tabular}{|l|ccc|ccc|ccc|}
\hline TYPE OF & \multicolumn{3}{|c|}{ \% Overshoot } & \multicolumn{3}{c|}{ Settling Time (sec) } & \multicolumn{3}{c|}{ Steady state error (pu) } \\
CONTROLLER & $\Delta \mathrm{Pm}_{1}$ & $\Delta \mathrm{Pm}_{2}$ & $\Delta \mathrm{P}_{12}$ & $\Delta \mathrm{Pm}_{1}$ & $\Delta \mathrm{Pm}_{2}$ & $\Delta \mathrm{P}_{12}$ & $\Delta \mathrm{Pm}_{1}$ & $\Delta \mathrm{Pm}_{2}$ & $\Delta \mathrm{P}_{12}$ \\
\hline Without controller & 25 & 5.3 & -5.2 & 20 & 10 & 10 & -0.1 & 0.055 & -0.055 \\
\hline With PI controller & 31 & 5.0 & -2.5 & 15 & 20 & 15 & 0.18 & 0.00 & 0.000 \\
\hline With Fuzzy controller & 14 & 5.0 & -7.0 & 12 & 12 & 12 & -0.15 & 0.05 & -0.05 \\
\hline
\end{tabular}

Table 2: 


\section{CONCLUSION}

Summary of performance indices the result shows that the fuzzy logic based LFC has superior performance characteristics as compared to the

\section{REFERENCES}

[1] Haadi Saadat, "Power Systems Analysis" McGrawHill companies Inc. 1999.

[2] Elgerd, O.I, "Electric Energy system theory: An Introduction" McGraw-Hill, TMH edition, 1971

[3] Jawat, T. and Fadel, A, B. "Adaptive Fuzzy Gain Scheduling for Load frequency control", IEEE Trans. on PAS, vol. 14, No 1, February 1999.

[4] Nanda, J. and Kaul, B.L, "Automatic Generation Control of an interconnected power system" IEE Proc. Vol. 125, No. 5, May 1978, pp 385-390.

[5] Fosha, C.E, and Elgerd, O.I. "The MegawattFrequency control problem: a new approach via optimal control theory, IEEE, Trans. pp 563-577, 1970.

[6] Edison, B, and Ilie, M, "Advanced Generation control: Technical Enhancements, costs, and Responses of market Driven Demand" Proc. of the $57^{\text {th }}$ Annual American Power conf; vol. 57, No. 2, 1995, pp 14191427.

[7] Gopal, M, "Modern control system theory" Wiley Eastern Ltd, $2^{\text {nd }}$ edition 1993.

[8] Alden, M, "A Fresh Approach to the LQR problem with Application to power systems" Proc. of int. power Engineering control, Singapore, Vol. 1, 1993, pp 374-379. conventional PI controller. Also that the supplementary control is highly needed to damp out the effect of load changes on system frequency and Tie-line interchange power flows.

[9] Elgerd, O.J, and Foshua, C.E "Optimum MegawattFrequency Control Problem of Multi-area Electric Energy Systems," IEEE Trans. on PAS, vol. PAS-89, No. 4, April 1970, pp 556-563.

[10] Kanniah, J, et al, "Microprocessor-Based Adaptive Load Frequency Control," IEE Proc; vol. 131, No. 4, July 1984.

[11] Vajik, L. et al, "Adaptive Load-Frequency Control of the Hungarian Power system," Automatica vol. 21, No. 2, 1985, pp 129-137.

[12] Chaturvedi, D.K, Satsangi, P.S, and Kalra, P.K; "Load frequency control: a generalized neural network approach" Electric Power and Energy systems, No. 21, 1999, pp 405-415.

[13] Hsu, Y, and Chang, C. "Load frequency control using Fuzzy logic Int. conf. on High Tech. in the power industry, 1991, pp 32-38.

[14] Indulkar, C.S, and Raj. B, "Application of fuzzy controller to Automatic Generation control, "Electric machines and power systems; vol. 23, No 2, MarchApril 1995. pp 32-38

[14] Anand, B. And Ebenezer, A.J., "Load Frequency control with fuzzy logic controller considering Nonlinearities and Boiler Dynamic" ICGST-ACSE Journal vol. 8, issue 111, Jan. 2009.

[15] Learning simulink, mathworks, Inc, 2001

[16] Passino K.M, and Yurkovich S., "Fuzzy control," Addison-Wesley 1998 